\title{
P18 Vascular Aging Index of the Finger Photoplethysmogram: A Validation Study with Vascular Stiffness, Mental Stress, and Day-to-Day Variability
}

\author{
Takashi Tarumi ${ }^{1 *}$, Takayuki Yamabe ${ }^{2}$, Jun Sugawara ${ }^{1}$ \\ ${ }^{1}$ National Institute of Advanced Industrial Science and Technology, Tsukuba, Japan \\ ${ }^{2}$ University of Tsukuba, Tsukuba, Japan
}

\begin{abstract}
Background: Daily assessment of vascular health may predict cardiovascular incidence. Vascular aging index (VAI) calculated from second derivative of photoplethysmogram (SDPTG) is a simple, non-invasive measure possibly reflecting vascular stiffness. However, the effects of daily life events such as mental stress and day-to-day variability as well as its relation to other indices of vascular stiffness remain unclear.

Purpose: To determine whether VAI measured by finger SDPTG is 1) correlated with peripheral augmentation index (pAI), 2) altered by acute mental stress, and 3) affected by day-to-day variability.

Methods: Simultaneous measurements of finger photoplethysmogram and radial artery tonometry were performed in 68 healthy subjects (age $=22-64$ years) of whom 31 subjects were further tested during a 30-second mental arithmetic and 10 subjects underwent day-to-day variability assessment for 5 consecutive days. VAI was calculated from a 20 -second segment of photoplethysmogram data [1].

Results: At rest, VAI was positively correlated with pAI $(r=0.62, p<0.001)$. During mental arithmetic, VAI increased when compared with the control condition $(p=0.032)$ whereas pAI did not show significant change. Five day measurements of VAI demonstrated the overall coefficient of variation of $21.1 \pm 13.7 \%$ across all subjects.

Conclusion: VAI calculated from the finger SDPTG is related to a measure of vascular stiffness and sensitive to mental stress with fair day-to-day variability. These findings suggest that VAI assessment needs to be performed at the quiet resting condition.

\section{REFERENCE}

[1] Takazawa K, Tanaka N, Fujita M, Matsuoka O, Saiki T, Aikawa M, et al. Assessment of vasoactive agents and vascular aging by the second derivative of photoplethysmogram waveform. Hypertension 1998;32:365-70.

(c) 2019 Association for Research into Arterial Structure and Physiology. Publishing services by Atlantis Press International B.V. This is an open access article distributed under the CC BY-NC 4.0 license (http://creativecommons.org/licenses/by-nc/4.0/).
\end{abstract}

